\title{
Estimation of Trust Value for Secure Information Sharing on Social Network
}

\author{
P.Krishnamoorthy, K. Izas Ahmed, M.Karthik Kumar, V.P Kishodh
}

\begin{abstract}
To give a community oriented way to deal with effective information partaking in OSN. To make limit dependent on which the client makes the last choice on information posting. A high limit demonstrates that the client has a generally low propensity to impart the information to other people, and just when the larger part of the included clients or clients that are profoundly trusted consent to post the information, the information can at long last be posted. By tuning the edge, the client can make an exchange off between information sharing and protection saving. To give programmed remark examination and remark blocking approach for secure picture sharing.
\end{abstract}

\section{INTRODUCTION}

The appearance of the Internet and online networking has radically changed all parts of our lives; how we work, devour, and impart While this has had extensive focal points for society by and large, the developing impact of the Internet and innovations has dependably been connected to worries for protection and the gathering and utilization of individual data. The dangers to singular protection through these advancements have been over and again recorded. Over the previous years, touchy individual information were more than once unlawfully Acquired and misused in various information ruptures. Most as of late, delicate individual data 150 million individuals was imperiled in the 2017 Equifax information rupture.

While a few buyers are ignorant of the information they produce or of the full degree to which their information are mined and investigate others couldn't care less. A dominant part of buyers, be that as it may, report worries about their online security and, yet, a great many people frequently exchange their own information for online administrations and items, For example, even protection concerned people join long range interpersonal communication administrations, for example, Facebook, and offer a lot of individual data on these stages.

Furthermore, mental systems hidden these valuations will be inspected. In the accompanying, the logical writing hidden this exploration will be evaluated and the examination speculations for this exploration will be created. The test structure and research techniques will be laid out and the foreseen outcomes exhibited and talked about.

Revised Manuscript Received on April 12, 2019.

P.Krishnamoorthy,Department of Computer Science and Engineering, V.S.B. Engineering College Karur, Tamil Nadu, India- 639111 (krishnancse0206@gmail.com)

K. Izas Ahmed,Department of Computer Science and Engineering, V.S.B. Engineering College Karur, Tamil Nadu, India- 639111

M.Karthik Kumar,Department of Computer Science and Engineering, V.S.B. Engineering College Karur, Tamil Nadu, India639111

V.P Kishodh,Department of Computer Science and Engineering, V.S.B. Engineering College Karur, Tamil Nadu, India- 639111

\section{RELATED WORKS}

\section{I.Profile Sharing}

A few OSNs can give open stages to empower any outsider engineers worldwide to make full applications on the highest point of clients' profiles, inside the structure. To empower social applications to be increasingly intentional and important, they may devour client profile properties, which more often than exclude data, for example, the client's name, birthdates, status, address, messages, instruction, interests, photographs, music, recordings, and numerous different qualities. In the meantime, outsider applications could be broadened and use traits of client's companions, which would present genuine protection worries for the companions. Whenever clients and their companions utilize a similar application, both the client and her/his companion need to control which traits the application can get to. Current OSNs permit just a single side of the relationship to administer access to the profile characteristics of the opposite side. Thus, the choice of a getting to an application is exclusively managed by the client who wants to share her/his companion data with an outsider application in the OSN. To address such a basic issue, we consider the client's companion is a proprietor who claims shared information on her/his space, which comprises of profile characteristics. The second controller is a giver who shares her/his companion's profile properties with a social application. At that point, we offer a component to consolidate proprietor and benefactor protection settings of the common profile traits. Our proposed arrangement, to be, with its fundamental sources of info, is appeared between the application and the information to be controlled.

\section{II.Relationship sharing}

Users in OSNs are connected by social relationships, which characteristically are bidirectional. OSNs enable users to share their relationships with other members in OSNs. In fact, there are two users who establish the relationship in OSNs; consequently, both of them have the right to manage who can see the relationship between them. However, current OSNs provide limited access control that allows only one side of a relationship to restrict access where the user on the other side of the relationship may have a different privacy preference. Consequently, the result of current OSN access control causes a high level of disclosure in online relationships because the participants in a relationship may have dissimilar sensitivity levels with respect to each other. 
The associated users in this case are co-owners. The need for a solution addressing the problem of relationship information leakage is demonstrated, where the relationship is the shared item between two users. The first user is called a stakeholder who specifies a policy to hide her/his relationships from the public. Thus second user is an owner who adopts a weaker policy that allows the public to see her/his relationships list. In this scenario, to regulate a satisfactory policy we have to consider owner and stakeholder authorization requirements to achieve a collective decision. Again, our proposal for handling this, Accessory, which is shown intervening between the accessory and the data.

\section{Content Sharing}

OSNs offer mechanisms that facilitate users to socialize in the digital world. The main purpose of relationships in OSNs is to share various resources, which includes information, photographs, music and videos. Posting, tagging and information exchange are sharing tools that are provided by many OSNs such as Facebook, Google+ and My Space. In this section, we introduce content sharing scenarios for which privacy policies in current OSNs do not adequately provide collective privacy controls on shared content. First, a user is able to post notes and news in her/his own space, upload pictures and videos, tag others members in her/his contents and share her/his contents such as pictures, videos, news etc., with other users. Furthermore, OSNs allow users to post contents on their friends' profiles and share their friends' contents. We organize the scenarios for content sharing depending on the sharing tools that are applied to the content.

\section{IV.Sharing}

The sharing tool supports distributing data among members in OSNs in various ways. Users can share their contents with others in their social network; otherwise, users can share others users' contents. Also, users can share other user's content and post it in someone else's space. In general, whenever types of sharing apply to an item in OSNs there is high potential of identification all linked users who are related to this item. Current OSNs, until now, provide individual processes to make a decision over who can access the shared items. As a result, those items, which obviously expose the identity of all associated users, may violate a users' privacy and lead to their embarrassment. We believe it could be more practical and reliable to allow all linked users to participate in the privacy setting of a shared item. To reach this goal, first we introduce and analyze three multiple controllers' scenarios that are raised by using the sharing tool, then we can solve the problem of how to merge privacy opinions from co-controllers of shared items.

\section{V.Proposed system}

The proposed theories plan to discover the effect of protection, security and trust on the ability to share data on person to person communication locales. As indicated by the proposed theories, saw security, saw protection and saw trust are the variables that impact a client's readiness to share data on informal communication locales.

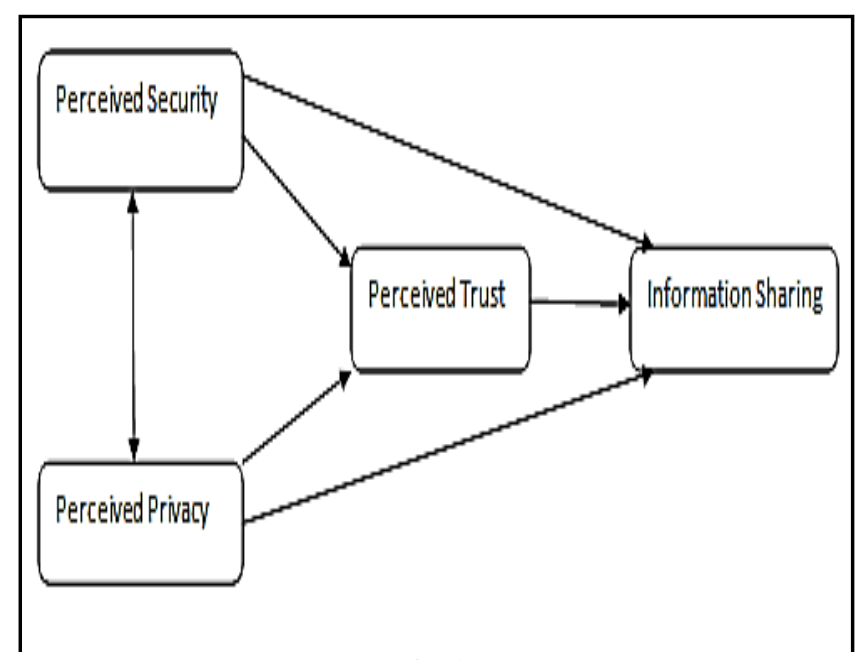

Fig 1

\section{VI.Privacy}

Privacy is characterized as a procedure of secrecy safeguarding and is emphatically associated with power over data about oneself. In online situations, individuals who see higher dangers to security are less arranged to uncover data about the self since they see themselves as less ready to control data and furthermore ensure themselves. Interestingly, when individuals see lower protection dangers and higher control, for example, when security approaches are plainly uncovered, they reveal increasingly close to home data.

\section{VII.Perceived Trust}

Trust is compulsory when chance is available. Trust is the establishment of any exchange that happens between two gatherings. There are a few features to the idea of trust. For eye to eye, trust is a basic determinant of sharing data and growing new connections.

\section{VIII.Perceived Security}

From a customer point of view, saw security of an electronic trade exchange might be characterized as 'the emotional likelihood with which buyers trust that their own data (private and financial) won't be seen, put away, and controlled amid travel and capacity by wrong gatherings in a way reliable with their certain desires'. Security compares to worries about the insurance of individual data with three explicit objectives: uprightness that guarantees data isn't modified amid travel and capacity; confirmation that tends to the check of a client's personality and qualification for information access; and secrecy that necessitates that information use be restricted to approved purposes by approved individuals.

\section{IX.Information Sharing}

In line with previous research, the data show high levels of information revelation on Facebook. A huge number of individuals have joined interpersonal interaction destinations, including profiles that uncover individual data. The notorieties of person to person communication locales have been lessened by various occurrences announced by the news media. 


\section{EXPERIMENTAL ANALYSIS}

\section{Control Increase trust}

The essential goal of the examination is to explore the impact of clients' protection worries on utilization conduct and data sharing on informal communication destinations with reference to Facebook. Human conduct issues have a significant job in the sending of interpersonal interaction locales. Utilizing the all-encompassing TAM and social trade hypothesis, the proposed research structure was experimentally tried. Our examination discoveries recommend that clients having power over their data stream and insurance of their profile is bound to prompt trust in Facebook. Another consider that influenced trust Facebook was the security highlights given by Facebook and individual conviction that getting to Facebook over the web is secure.

\section{II.Privacy and sharing}

Further results suggest that perceived privacy and perceived security are antecedents of perceived trust, whereas there is a strong correlation between perceived privacy and perceived trust. As far as data sharing, when trust is applied through protection and trust, this prompts the client eagerness to share data. Conversely, security has no immediate impact on the sharing of data - an intriguing consequence of the investigation. This demonstrates trust in the capacity of Facebook will prompt a propensity for clients to share more data.

\section{III.Practical implication for site operators}

Beside hypothetical qualities, the outcomes have noteworthy reasonable ramifications. The discoveries may furnish interpersonal organization administrators with a superior comprehension of how security concerns may influence client acknowledgment and data disclosure. This examination gives an understanding to site administrators into clients' feeling of having a place as a thought process in sharing data and clients' security concerns, which may lead administrators to create and advance relating applications. There are sure restrictions to this investigation, in any case. To start with, a large portion of the exploration respondents had a place with the age gathering of 16- 35 years, which may not cover the overall public of person to person communication locales clients. Second, interpersonal organization clients lived in various nations, were from various societies and had diverse view of protection concerns, which possibly impacted their utilization conduct.

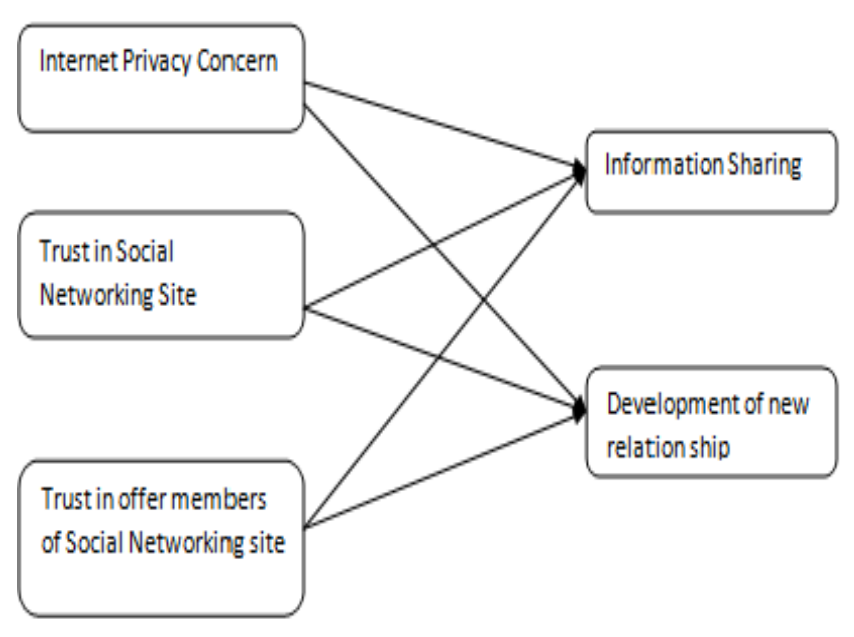

Fig 2

\section{IV.Pilot and reliability tests}

Information was gathered by directing an online tweaked poll study of Facebook clients to test the proposed system. The study was led from May to August 2013. To augment the reaction rate, we used web crawlers and messages. All things of the proposed research structure were estimated on a 5-point Likert scale, running from 1 (emphatically deviate) to 5 (concur). A pilot overview was utilized for approving the proposed structure before the last examination. Dependability examination was performed utilizing SPSS 19.0. Cronbach's alpha $(\alpha)$, which gives a proportion of the inward consistency of a test or scale, was utilized to test the inside consistency of the survey. SEM (auxiliary condition displaying) was utilized to recognize connections among develops. SEM was performed utilizing AMOS 19.0.

\section{V.Demographic profile of respondents}

The online poll was conveyed through messages and web search tools. An aggregate of 265 surveys were gathered over the time span of 4 months, out of which 246 were usable for the examination.

\section{VI.Reliability and Validity analysis}

Before examining the examination system, dependability investigation was utilized to test the interior consistency of the poll. Cronbach's $\alpha$ is a broadly utilized estimation for inside consistency. To guarantee the unwavering quality of the investigation, things were adjusted dependent on a worthy Cronbach's $\alpha$ score above 0.60 , in view of standard qualities.

\section{VII.Model fit summary}

Corroborative factor examination is utilized for the model attack of the proposed system. For auxiliary condition display fit, different fit records and tests have been created. These files and tests, be that as it may, can point to decisions about the degree to which a model really coordinates the watched information, known as great model fit including non-trial look into. 


\section{IX.Trust Value Estimation}

The filtering rules should allow users to state constraints on message creators. Thus, creators on which a filtering rule applies should be selected on the basis of several different criteria. Filtering rules identifying messages according to constraints on their contents. It block the users who are post the negative comments more than five times and also send mobile intimation to users at the time offline.

\section{X.Comment Analysis}

Comment analysis working on the basis of image sharing and comment blocking process. Every posted images are shown to the other users, then users can set comment for particular image. Comment are compared with dataset to identify the positive and negative set. Filtering approach is used to analyze comment set.

\section{XI.Filter Approach}

The filtering rules should allow users to state constraints on message creators. Thus, creators on which a filtering rule applies should be selected on the basis of several different criteria. Filtering rules identifying messages according to constraints on their contents. It block the users who are post the negative comments more than five times and also send mobile intimation to users at the time offline.

\section{CONCLUSION}

In this paper we study the privacy issue caused by the sharing of co-owned data in OSNs. To help the owner of data collaborate with the stakeholders on the control of data sharing, we propose a trust-based mechanism. When a user is about to post a data item, the user first solicits the stakeholders' opinions on data sharing, and then makes the final decision by comparing the aggregated opinion with a pre-specifiedthreshold. The more the user trusts a stakeholder, the more the user values the stakeholder's opinion. If a user suffers a privacy loss because of the data sharing behavior of another user, then the user's trust in another user decreases. On the otherhand, considering that the user needs to balance between data sharing and privacy preserving, we apply a bandit approach totune the threshold in the proposed trust-based mechanism, so that the user can get a high long-turn payoff which is definedas the difference between the benefit from posting data and the privacy loss caused by other users. We have conductedsimulations on synthetic data and real-world data to verify the feasibility of the proposed methods. Simulation results showthat compared to directly posting data without asking others for permission, a user will suffer less privacy loss if he/shealways considers other users' privacy. And by applying the proposed UCB policy to determine the threshold, the user can get higher payoffs than setting the threshold to a fixed or random value.

\section{RESULT ANALYSIS}

Facebook than MySpace. For instance, the Facebook mean for "I believe that [SNS] won't utilize my own data for some other reason for existing" is 4.971, while the MySpace mean is lower, 4.396. The two noteworthy contrasts, and two others that help similar discoveries, demonstrate the dimension of trust in Facebook is higher than the dimension of trust in MySpace. This is a powerless unwavering quality outcome, anyway for new research results as low as .50 are satisfactory, despite the fact that an increasingly settled esteem is .7 (Goodhue, Klein, and March, 2000). In this way these two inquiries will be joined for factual examination. Individuals from Facebook show altogether higher trust in the site dependent on the joined measures (Facebook mean is 8.8382 , MySpace is $7.6875, \mathrm{~F}=4.511, \mathrm{p}=.036$ ). The two inquiries identified with trust in different individuals don't have a satisfactory unwavering quality, and will be treated as discrete measures.

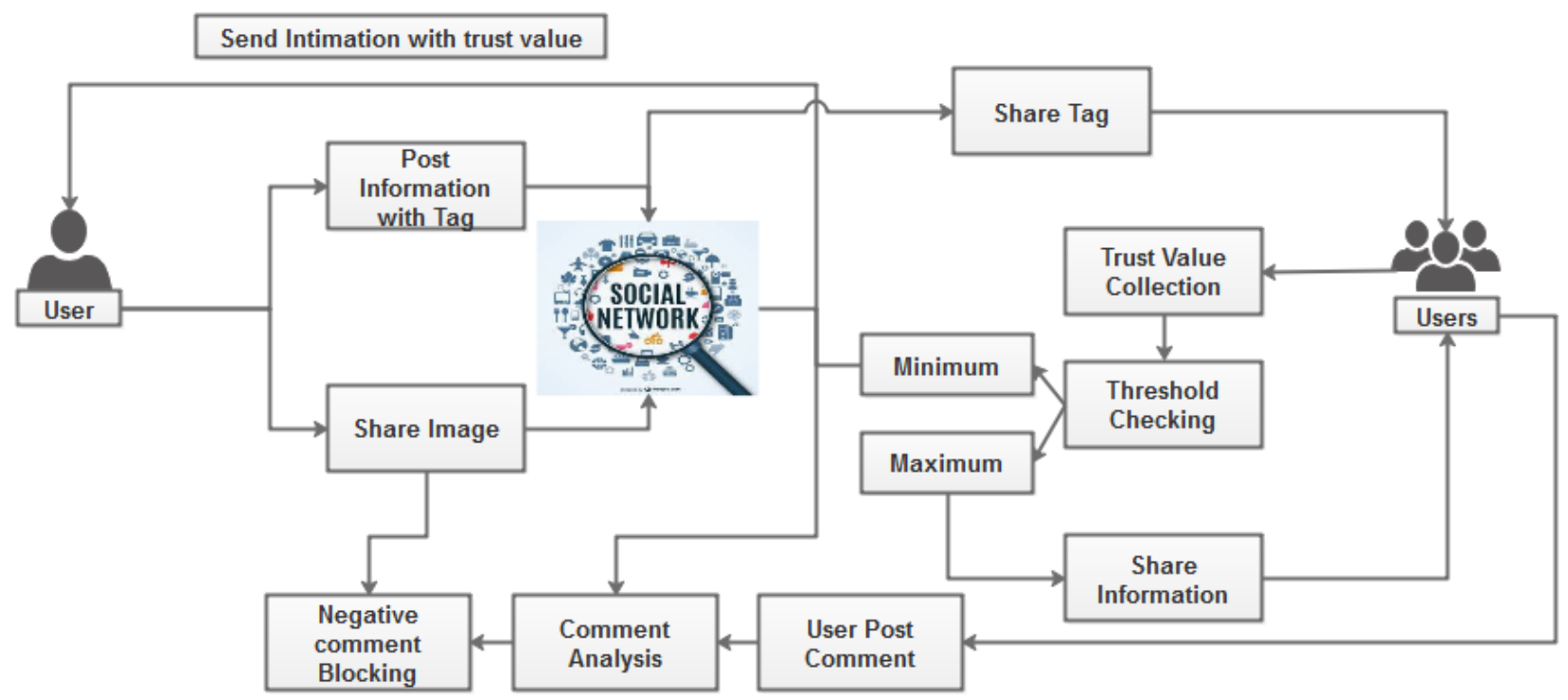

Fig 3 


\section{REFERENCE}

1. C. Zhang, J. Sun, X. Zhu, and Y. Fang, "Privacy and security for online social networks: challenges and opportunities," IEEE Network, vol. 24, no. 4, pp. 13-18, July 2010.

2. L. Xu, C. Jiang, J. Wang, J. Yuan, and Y. Ren, "Information security inbig data: Privacy and data mining," IEEE Access, vol. 2, pp. 1149-1176,2014.

3. L. Xu, C. Jiang, Y. Chen, J. Wang, and Y. Ren, "A framework for categorizing and applying privacy-preservation techniques in big data mining," Computer, vol. 49, no. 2, pp. 54-62, Feb 2016.

4. M. Qiu, K. Gai, and Z. Xiong, "Privacy-preserving wireless communications using bipartite matching in social big data,"Future Generation Computer Systems, 2017. [Online].Available:http://www.sciencedirect.com/science/artic le/pii/S0167739X1730149

5. C. Fiesler, M. Dye, J. L. Feuston, C. Hiruncharoenvate, C. Hutto,S. Morrison, P. KhanipourRoshan, U. Pavalanathan, A. S. Bruckman,M. De Choudhury, and E. Gilbert, "What (or who) is public?: Privacysettings and social media content sharing," in Proceedings of the 2017ACM Conference on Computer Supported Cooperative Work and SocialComputing, March 2017, pp. 567-580.

6. A. C. Squicciarini, M. Shehab, and F. Paci, "Collective privacy managementin social networks," in Proceedings of the 18th ACM InternationalConference on World Wide Web, April 2009, pp. 521-530.

7. H. Hu, G.-J. Ahn, and J. Jorgensen, "Detecting and resolving privacyconflicts for collaborative data sharing in online social networks," inProceedings of the 27th ACM Annual Computer Security ApplicationsConference, December 2011, pp. 103112.

8. J. M. Such and N. Criado, "Resolving multi-party privacy conflicts insocial media," IEEE Transactions on Knowledge and Data Engineering,vol. 28, no. 7, pp. 1851-1863, July 2016.

9. P. Auer, N. Cesa-Bianchi, and P. Fischer, "Finite-time analysis of themultiarmed bandit problem," Machine learning, vol. 47, no. 2-3, pp.235-256, 2002.

10. H. Hu, G.-J. Ahn, Z. Zhao, and D. Yang, "Game theoretic analysis ofmultiparty access control in online social networks," in Proceedings ofthe 19th ACM Symposium on Access Control Models and Technologies,New York, NY, June 2014, pp. 93-102.

11. H. Hu, G. J. Ahn, and J. Jorgensen, "Multiparty access control foronline social networks: Model and mechanisms," IEEE Transactions onKnowledge and Data Engineering, vol. 25, no. 7, pp. 1614-1627, July2013.

12. N. Vishwamitra, Y. Li, K. Wang, H. Hu, K. Caine, and G.-J. Ahn, "Towards pii-based multiparty access control for photo sharing in onlinesocial networks," in Proceedings of the $22 \mathrm{Nd}$ ACM on Symposium onAccess Control Models and Technologies, June 2017, pp. 155-166.

13. P. Mehregan and P. W. Fong, "Policy negotiation for coowned resourcesin relationship-based access control," in Proceedings of the 21st ACMon Symposium on Access Control Models and Technologies, June 2016,pp. 125-136.

14. J. Golbeck, "Trust on the world wide web: A survey," Foundations andTrends in Web Science, vol. 1, no. 2, pp. 131-197, 2008.

15. S. Zakhary, M. Radenkovic, and A. Benslimane, "Efficient locationprivacy-aware forwarding in opportunistic mobile networks," IEEETransactions on Vehicular Technology, vol. 63, no. 2, pp. 893-906,February 2014.

16. S. Zakhary and A. Benslimane, "On location-privacy inopportunistic mobile networks, a survey," Journal of Networkand Computer Applications, 2017. [Online]. Available:

17. S. Xu, X. Li, T. P. Parker, and X. Wang, "Exploiting trustbased socialnetworks for distributed protection of sensitive data," IEEE Transactionson Information Forensics and Security, vol. 6, no. 1, pp. 39-52, March2011.

18. N. Z. Gong and D. Wang, "On the security of trustee-based social authentications," IEEE Transactions on Information Forensics and Security, vol. 9, no. 8, pp. 1251-1263, Aug 2014.

19. W. Sherchan, S. Nepal, and C. Paris, "A survey of trust in social networks," ACM Computing Surveys, vol. 45, no. 4, pp. 47:1-47:33, August 2013.

20. Y. Tang, H. Wang, and W. Dou, "Trust based incentive in $\mathrm{p} 2 \mathrm{p}$ network,"in IEEE International Conference on E-Commerce Technology for Dynamic E-Business, September 2004, pp. 302-305.

21. N. C. Rathore and S. Tripathy, "A trust-based collaborative access control model with policy aggregation for online social networks," Social Network Analysis and Mining, vol. 7, no. 1, p. 7, February 2017.

22. Y. Sun, C. Zhang, J. Pang, B. Alcalde, and S. Mauw, "A trustaugmentedvoting scheme for collaborative privacy management," J. Comput. Secur.,vol. 20, no. 4, pp. 437-459, July 2012.

23. V. Buskens, "The social structure of trust," Social Networks, vol. 20,no. 3, pp. 265-289, 1998.

24. S. Nepal, W. Sherchan, and C. Paris, "Strust: A trust model forsocial networks," in 2011 IEEE 10th International Conference on Trust,Security and Privacy in Computing and Communications, November2011, pp. 841-846. 